\title{
Development of a Web-Based Decision Support System for Cell Formation Problems Considering Alternative Process Routings and Machine Sequences
}

\section{Chin-Chih Chang}

Department of Information Management, Jen-Teh Junior College of Medicine, Nursing and Management, Taiwan, China. Email: chinju.chang@gmail.com

Received November $16^{\text {th }}, 2009$; revised December $3^{\text {rd }}, 2009$; accepted December $12^{\text {th }}, 2009$

\begin{abstract}
In this study, we use the respective advantages of the tabu search (TS) and the Web-based technologies to develop a Web-based decision support system (DSS) for cell formation (CF) problems considering alternative process routings and machine sequences simultaneously. With the assistance of our developed Web-based system, the CF practitioners in the production departments can interact with the systems without knowing the details of algorithms and can get the best machine cells and part families with minimize the total intercellular movement wherever and whenever they may need it. To further verify the feasibility and effectiveness of the system developed, an example taken from the literature is adopted for illustrational purpose. Moreover, a set of test problems with various sizes drawn from the literature is used to test the performance of the proposed system. Corresponding results are compared to several well-known algorithms previously published. The results indicate that the proposed system improves the best results found in the literature for $67 \%$ of the test problems. These show that the proposed system should thus be useful to both practitioners and researchers.
\end{abstract}

Keywords: Web-Based, Cell Formation, Tabu Search, Decision Support System, Alternative Process Routings

\section{Introduction}

In response to various and diversified customer demands, companies must adopt innovative manufacturing strategies and manufacturing technologies to achieve an efficient and flexible manufacturing system. Group technology (GT) is one such approach that meets the requirements of system flexibility and product variations. GT is a manufacturing philosophy, which determines and divides the components into families and the machines into cells by taking advantage of part similarity in processing and design functions. Studies show that 30\%-75\% of the product cost is due to materials handling [1]. Cellular manufacturing (CM) is the application of group technology (GT) in manufacturing systems. The implementation of cellular manufacturing system (CMS) design has been reported to result in significant benefits such as reductions in material handling costs, work-in-progress inventory, throughput times and set-up times, simplified scheduling and improved quality [2]. Cell formation (CF) is the crucial element in designing CMS. However, it has been known that the CF in CMS is one of the NP-hard combinational problems [3], as it becomes difficult to obtain optimal solutions in an acceptable amount of time, especially for large-sized problems. While considering alternative process routings and machine sequences to CF problems, making the problems more realistic; however, it leads to a more complex problem than the simple CF problem. Thus, development of an effective computer-aided manufacturing CF support system is necessary. In this regard, many models and solution approaches have been developed to identify machine cells and part families. These approaches can be classified into three main categories [4]: 1) mathematical programming (MP) models [5-8], 2) heuristic/meta-heuristic solution algorithms [9-11], and 3) similarity coefficient methods (SCM) [12,13].

Due to their excellent performance in solving combinatorial optimization problems, meta-heuristic algorithms such as genetic algorithm (GA), simulated annealing (SA) and tabu search (TS) have been the most successful solution approach to efficiently solve the CF problem and its variants with good results. Among the meta-heuristic algorithms, TS has been successfully used to solve many problems appeared in manufacturing system including cell formation problems [14]. Hence, we adopt it as a solver to solve the $\mathrm{CF}$ problem considering alternative 
process routings and machine sequences simultaneously in the development of our CF Web-based decision support system (DSS).

In addition, CF algorithms are usually expressed in mathematical terms, which may only be understood by domain experts, but not by most of the CF practitioners in the production departments. With the assistance of CF DSS, users can interact with the systems without knowing the details of algorithms. Moreover, due to an increasing global competition, companies are now shifting to a geographically distributed manufacturing environment. Besides, the information flow nowadays requires reliability, efficiency and security. With the emergence of information technology, the traditional way of communication of information flows between companies and between internal parties can now be replaced by the interconnected network [15]. The Internet provides an open environment for companies to connect with their business partners as well as to serve as a medium for internal information flows [16]. Moreover, manufacturing systems have migrated to integrate with the Internet to provide a remote access and control system with the characteristics of quick response and real line monitoring [17].

In this study, we use the respective advantages of the TS and the Web-based technologies to develop a Webbased DSS for CF problem considering alternative process routings and machine sequences simultaneously. An example taken from the literature is adopted for illustrational purpose. To further verify the feasibility and effectiveness of the system developed, ten test problems with various sizes drawn from the literature are used to test the performance of our proposed CF solver. Corresponding results are compared to several well-known algorithms previously published.

The remainder of this article is organized as follows: Section 2 describes the problem definition including cell formation and the mathematical model; Section 3 details the implementation of our Web-based CF DSS; Section 4 verifies the performance of the proposed system and methodology; and Section 5 concludes the paper.

\section{Problem Definition}

\subsection{Cell Formation}

In a simple CF problem, cell formation in a given 0-1 machine-part incidence matrix involves rearrangement of rows and columns of the matrix to create part families and machines cells, in which the cellular movement can be minimized and the utilization of the machines within a cell can be maximized. Two matrices shown in Figure 1 are used to illustrate the concept. Figure 1(a) is an initial matrix where no blocks can be observed directly. After rearrangement of rows and columns, two blocks can be obtained along the diagonal of the solution matrix in Figure 1(b). For those 1's outside the diagonal blocks, they are called "exceptional elements"; while those 0's inside the diagonal blocks are called "voids".

When parts have alternative process routings (APR) is called the generalized CF problem. Such as the case shown in Table 1, part \#1 has two process routings R1 and R2. Kusiak [5] first described the problem and presented an integer-programming model to solve the problem. While introducing APR to CF problems, the grouping of parts can be more effective due to the flexibility of the routes; however, it leads to a more complex problem than the simple CF problem. Under this circumstance, not only the formation of part families and machine cells must be determined but also the selection of routings for each part has to be determined to achieve decision objectives such as the minimization of intercellular movement. For instance, Table 2 provides a feasible solution to the sample problem of Table 1 with a total intercellular movement of 215

\subsection{Mathematical Model}

The decision objective of their research is to minimize the sum of total intercellular movement. The $0-1$ integer programming model that they formulated is given below, and the notations are introduced first.

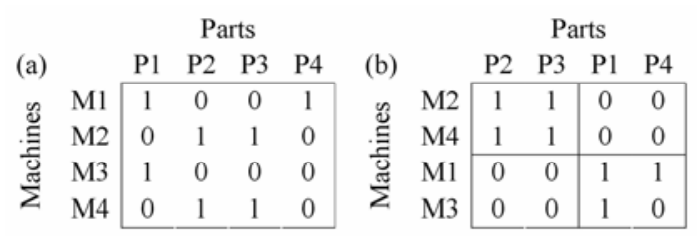

Figure 1. Rearrangement of rows and columns of matrix to create cells: (a) initial matrix and (b) matrix after rearrangement

Table 1. Initial machine-part matrix where alternative process routings are allowed

\begin{tabular}{|c|c|c|c|c|c|c|c|c|c|c|c|c|c|c|c|c|}
\hline PV & 7 & & 13 & & 11 & & 1 & & 11 & & 10 & & 14 & & 11 & \\
\hline$\overline{\mathrm{PN}}$ & $\bar{P}$ & & $\overline{\mathrm{P}^{2}}$ & & $\overline{\mathrm{P}}$ & & $\overline{\mathrm{P}}$ & & $\bar{P}$ & & $\vec{P}$ & & $\mathrm{P}^{\prime}$ & & P8 & \\
\hline $\mathrm{RN}$ & R1 & & R1 & 22 & R1 & R2 & $\mathrm{R} 1$ & R2 & $\mathrm{R} 1$ & $\bar{R} 2$ & R11 & R2 & & $\mathrm{R}$ & RI & $\overline{\mathrm{R} 2}$ \\
\hline$\overline{\mathrm{Ml}}$ & *1 & & 1 & & 1 & 1 & 1 & 1 & & 1 & & 1 & 1 & & 1 & \\
\hline $\mathrm{M} 2$ & & 1 & & 1 & & & & & 1 & & 1 & & & 1 & & 1 \\
\hline M3 & & & & & 2 & 2 & 2 & 2 & & & & & & & & \\
\hline M4 & 2 & & 2 & & & 3 & 3 & 3 & & 2 & & & 2 & & & \\
\hline M5 & 3 & & 3 & 2 & 3 & & & 4 & & & & & 3 & & & \\
\hline M6 & & 2 & & & & & & & & & & & & 2 & 2 & 2 \\
\hline $\mathrm{M} 7$ & & & & & & & & & 2 & 3 & 2 & 2 & & & & \\
\hline M8 & & 3 & 4 & & 4 & 4 & 4 & 5 & & & 3 & 3 & & & & \\
\hline MO & 4 & & & 3 & & & & & & 4 & & & 4 & 3 & & \\
\hline
\end{tabular}

PV: Production Volume; PN: Part Number; RN: Routing Number; * Process Sequence 
P5 P6 P8 P1 P2 P3 P4 P7

R1 R1 R2 R1 R1 R1 R1 R1

\begin{tabular}{l|l:l:l|l|l|l|l|l|} 
M2 & 1 & 1 & 1 & & & & & \\
M6 & & & 2 & & & & & \\
M7 & 2 & 2 & & & & & & \\
\hline M1 & & & & 1 & 1 & 1 & 1 & 1 \\
M3 & & & & & & 2 & 2 & \\
M4 & & & 2 & 2 & & 3 & 2 \\
M5 & & & 3 & 3 & 3 & & 3 \\
M8 & & 3 & & & 4 & 4 & 4 & \\
M9 & 3 & & & 4 & & & 4 \\
\hline
\end{tabular}

Figure 2. Final machine-part matrix of Table 1

Notations:

$\begin{array}{cl}m & \text { Number of machines } \\ p & \text { Number of parts } \\ N C & \text { Number of cells } \\ V_{i} & \text { Production volume for part } i \\ Q_{i} & \text { Number of routings for part } i \\ U_{m} & \text { Maximum number of machines in each cell } \\ L_{m} & \text { Minimum number of machines in each cell } \\ & \begin{array}{l}\text { Number of operations in routing } j \text { of part } i \text {; } \\ \text { the operations of part } i \text { along route } j \text { are processed }\end{array} \\ K_{i j} & \text { on a machines' set of } \\ & \left\{u_{i j}^{(1)}, u_{i j}^{(2)}, \ldots, u_{i j}^{(k)}, u_{i j}^{(k+1)}, \ldots, u_{i j}^{\left(K_{i j}-1\right)}, u_{i j}^{\left(K_{i j}\right)}\right\} \\ u_{i j}^{(k)} & \begin{array}{l}\text { Machine index for the } k \text {-th operation of part } i \text { along } \\ Y_{k l}\end{array} \\ Z_{i j} & 1, \text { if machine } k \text { locates in cell } l ; 0 \text {, otherwise } \\ & 1, \text { if routing } j \text { of part } i \text { selected; } 0, \text { otherwise }\end{array}$

The $0-1$ integer programming model is as follows:

$$
\text { Min } I C M=\sum_{i=1}^{p} \sum_{j=1}^{Q_{i}} \sum_{k=1}^{K_{i j}-1} \sum_{l=1}^{N C} V_{i} Z_{i j l} Y_{\left(u_{i j}^{(k)}\right) l}\left(1-Y_{\left(u_{i j}^{(k+1)}\right) l}\right)
$$

st:

$$
\begin{gathered}
\sum_{j=1}^{Q_{i}} Z_{i j}=1 \quad \forall i \in\{1,2, \ldots, p\} \\
\sum_{l=1}^{N C} Y_{k l}=1 \quad \forall k \in\{1,2, \ldots, m\} \\
L_{m} \leq \sum_{k=1}^{m} Y_{k l} \leq U_{m} \quad \forall l \in\{1,2, \ldots, N C\} \\
Y_{k l}, Z_{i j}, \in\{0,1\} \quad \forall i, j, k, l
\end{gathered}
$$

In the above model, Equations (1) is the objective function, which show the calculation of the total intercellular movement. Equation (2) indicates that only one process routing will be assigned to each part. Equation (3) provides a restriction that each machine will be assigned to exactly one cell. Equation (4) assigns the upper and lower limits of the cell size. Equation (5) indicates that
$Y_{k l}$ and $Z_{i j}$ are $0-1$ binary decision variables.

The large number of binary variables and constraints makes it difficult to obtain optimal solutions in an acceptable amount of time. Developing an effective computer-aided manufacturing CF support system is more appropriate than using the exact method in terms of solution efficiency, especially for large-sized problems. This paper, thus, presents an efficient Web-based DSS for CF problem. The developed system is described and explained in detail in the next section.

\section{System Development}

In this study, we dedicated to develop a Web-based CF DSS. With this system, the users can upload the CF data to Web serve and then with the CF solver executed, they can get the best machine cells and part families with maximize grouping efficacy wherever and whenever they may need it. The system architecture for CF DSS is shown in Figure 3. From the figure, we can know that the system consists of five elements. They are the clients (i.e., users), the Web-based user interface, the Web server, the CF solver and the database. All of them are linked up with the Internet but may be located in different geographical places. We will describe them below.

\subsection{Client and Web-based User Interface}

Web browsers are clients that connect to Web servers and retrieve Web pages for display. Using appropriate Web browsers, such as Netscape's Navigator or Microsoft's Internet Explorer, users can input data or view the CF results through a dynamic hypertext user interface. Because of the PHP is a widely-used general-purpose scripting language that is especially suited for Web development and can be embedded into HTML. Hence, we use PHP to making dynamic and interactive Web pages for the Web-based user interface which consists of three buttons on the top of the screen. The framework of the user interface is shown in Figure 4 which is simple and Framework of Web-based user interface

Upload Data: The client users can upload production data to Web serve by using this button. The production

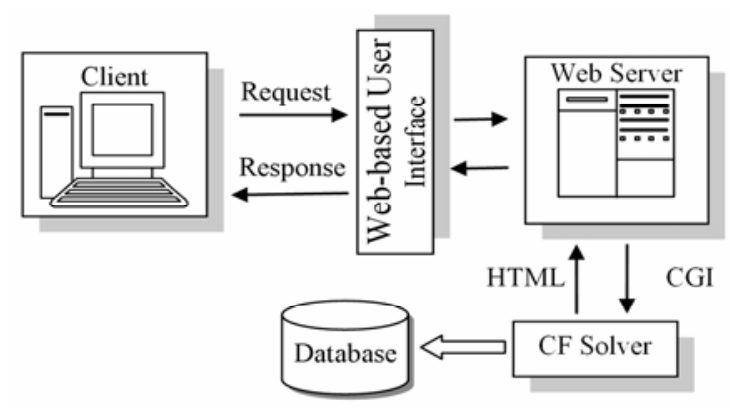

Figure 3. System architecture for CF DSS 


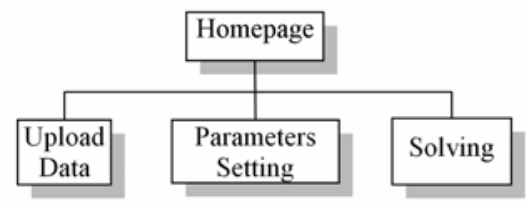

Figure 4. Considered to be user-friendly, we will describe them below

data are given through a text document readable with any text viewer $(*$.txt).

Parameters Setting: The client users can set up the parameters for $\mathrm{CF}$ solver.

Solving: With this button be pressed, the CF solver will be executed to minimize the total intercellular movement.

\subsection{Web Server}

The Web server is a computer that serves requested Web pages. The Web server interacts with the individual user's Web browser and accepts external Hypertext Transfer Protocol (HTTP) requests from the browser. An Application Programmer's Interface (API) is distributed, along with most of the commercially available browsers, such as Netscape's Navigator or Microsoft's Internet Explorer. Application programs, such as PHP, ASP and JSP, can be written using these APIs to enhance the capabilities of a browser. Because of the Apache HTTP Server has been the most popular Web server on the Internet since April 1996. Therefore, we use the Apache server as Web server in this study.

\subsection{CF Solver}

The CF solver was developed using Visual C++ programming languages. It consists of two stages: the initial solution construction and the improvements. The Single Linkage Clustering Algorithm (SLCA) of McAuley [13] is adapted in the first stage to produce good initial solutions, while the TS continuously improves and generates more effective solutions through the TS algorithm in the second stage. The proposed generic framework for the CF solver is shown in Figure 5 which is actually consists of the following seven steps:

1) Initialization of computational parameters;

2) Construction of initial solution;

3) Searching of improving neighborhood solutions;

4) Update of tabu list;

5) Update of better solutions found;

6) Check of timing for directing searching toward diversified solution space by applying mutation operator;

7) Check of solution stagnancy.

Note that the first five steps are the same as the TS algorithm, while Step 6 generates new solutions with higher degree of diversification in order to increase the probability of finding the global optima, and Step 7 avoids spending too much computational efforts in order to have a balance between the computational effectiveness and efficiency.

For the CF solver, the insertion strategy is applied to produce a new neighborhood solution and the values of parameters are given below: tabu list size is equal to 7 , a limit of iterations for each run is set to 1000 and the mutation probability for each gene is set at 0.8 .

\subsection{Database}

A database server machine may be physically different from the Web server that maintains the database. Due to the MySQL is the world's most popular open source database. Hence, we use MySQL server as the databaseserver in this study. This remote database is accessed

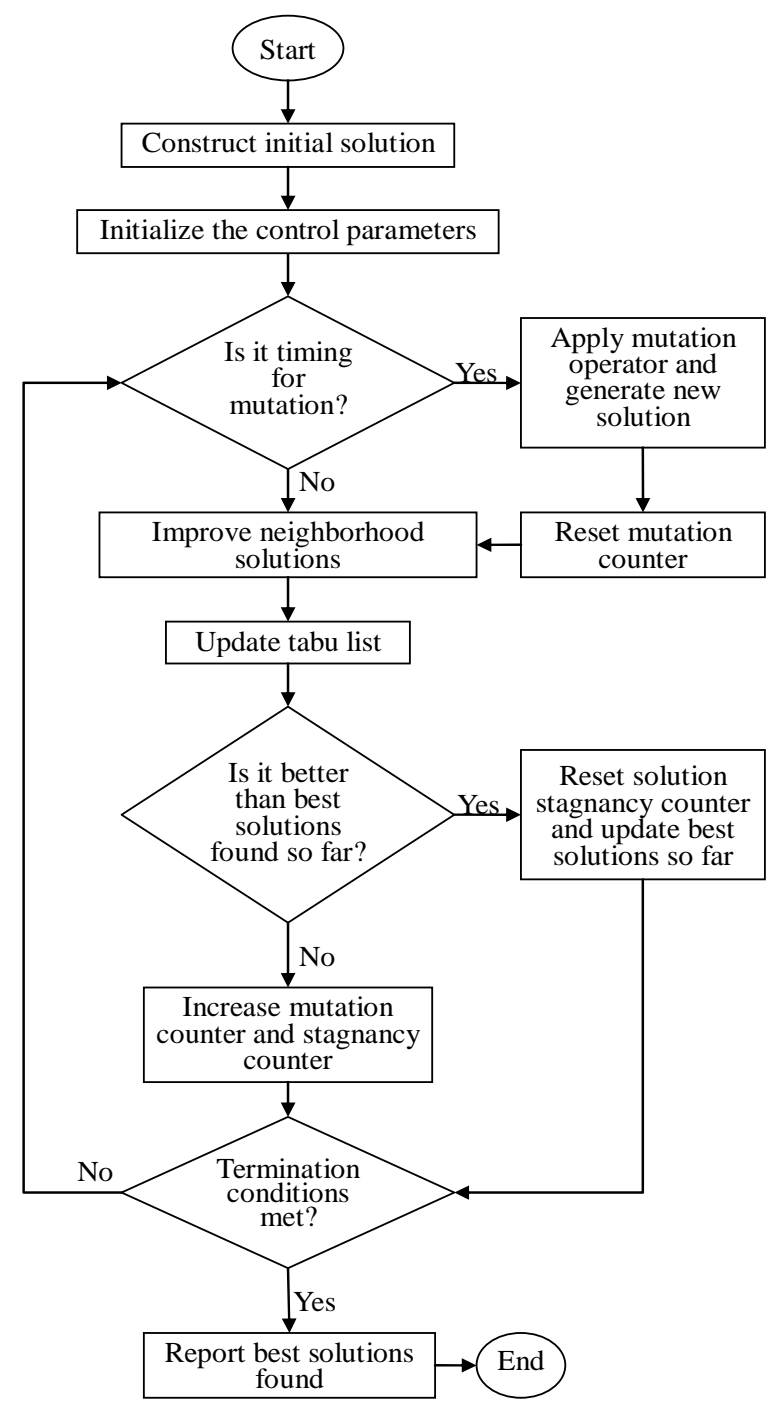

Figure 5. Flowchart of CF solver 
through the Open Database Connectivity (ODBC) gateway to insert, delete or update information in the database.

\section{Research Results}

The research results consist of two parts. They are the numerical illustration and the comparative study. We will describe them below.

\subsection{Numerical Illustration}

We applied a numerical example, which was drawn from Sofianopoulou [9], to test the performance and usability of our developed system. The step-by-step procedures are described as follows:

Step 1: Press the "Upload Data" button to upload the production data to Web server, as shown in Table 2, which consists of 4 machines and 5 parts.

Step 2: Set the parameters and constrains for CF solver by pressing the "Parameters Setting" button (See Figure 6).

Step 3: Press the "Solving" button to execute the CF solver to groups the machines into machine cells and parts into part families with minimize the total interce-

Table 2. Production data for the numerical example

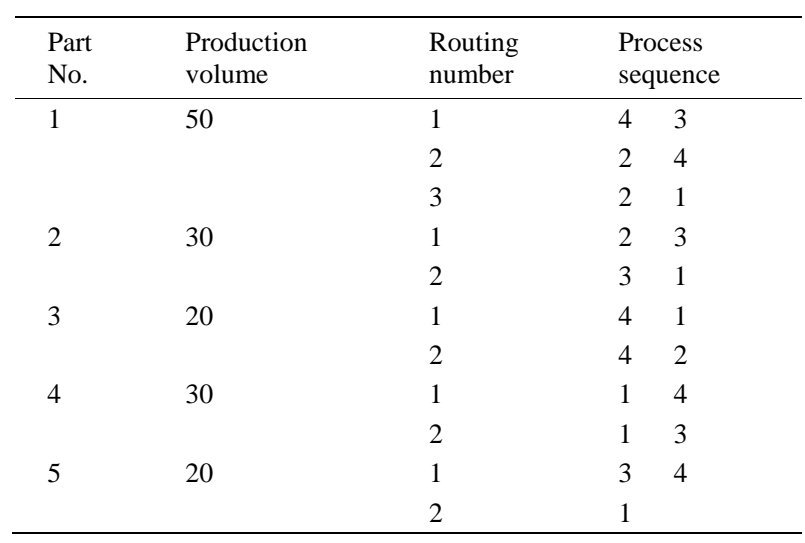

\begin{tabular}{|c|c|c|c|c|c|}
\hline & Upload Data & \multicolumn{3}{|c|}{$\mid$ Parameters Setting $\mid$} & Solving \\
\hline \multicolumn{6}{|c|}{ TS Parameters } \\
\hline \multicolumn{2}{|r|}{ Iteration } & \multicolumn{3}{|c|}{ Tabu List Size } & Mutation Probability \\
\hline & 1000 & & 7 & & 0.8 \\
\hline \multicolumn{6}{|c|}{ Test instances selection \& Constrains Setting } \\
\hline No. & Test Instances & $\mathrm{Y} / \mathrm{N}$ & $U_{m}$ & $L_{\mathrm{m}}$ & Number of cells \\
\hline 1 & P4_5.txt & 听 & 2 & 2 & Free \\
\hline
\end{tabular}

Figure 6. Web-based input interface for setting parameters and constrains

\begin{tabular}{|l|c|c|c|c|c|}
\hline \multirow{2}{*}{$\begin{array}{l}\text { Part } \\
\text { (PV) }\end{array}$} & \multirow{2}{*}{ Routing } & \multicolumn{4}{|c|}{ Maching } \\
\hline & & 2 & 4 & 1 & 3 \\
\hline $1(50)$ & 2 & 1 & 2 & & \\
\hline $3(20)$ & 2 & 2 & 1 & & \\
\hline $2(30)$ & 2 & & & 2 & 1 \\
\hline $4(30)$ & 2 & & & 1 & 2 \\
\hline $5(20)$ & 2 & & & 1 & \\
\hline Number of cells(NC) & & 2 \\
\hline Inter-Cell Movements(ICM) & 0 \\
\hline CPU(s) & & 0.015 \\
\hline
\end{tabular}

Figure 7. Web-based output interface for displaying CF results

Table 3. Test Instances From The Literature

\begin{tabular}{ccc}
\hline No. & Source & Size $(m \times p \times r)$ \\
\hline 1 & Nair and Narendran [12] & $8 \times 20 \times 20$ \\
2 & Sofianopoulou [9] & $12 \times 20 \times 26$ \\
3 & Wu [18] & $13 \times 13 \times 13$ \\
4 & Sofianopoulou [9] & $14 \times 20 \times 45$ \\
5 & Gupta and Seifoddini [19] & $16 \times 43 \times 43$ \\
6 & Sofianopoulou [9] & $18 \times 30 \times 59$ \\
7 & Harhalakis et al. [10] & $20 \times 20 \times 20$ \\
8 & Nagi et al. [20] & $20 \times 51 \times 51$ \\
9 & Nair and Narendran[12] & $25 \times 40 \times 40$ \\
\hline
\end{tabular}

llular movement. As shown in Figure 7, the CF solver only took 0.015 seconds to get the final configuration for the cell formation with two cells and without any inter-cell movement.

\subsection{Comparative Study}

In order to evaluate the computational characteristics of our proposed CF solver with other approaches, ten test instances from the literature, as shown in Table 3 were used. The proposed CF solver was coded in Visual C++ programming languages and implemented on an Intel(R) $1.66 \mathrm{GHz}$ personal computer with 1GB RAM. Table 4 shows the comparisons of our proposed CF solver with other approaches from the literature, that is, the GABB [10], the MIP [8] and the SA [9]. The bold characters indicate the best values obtained for each test problem. It can be seen from Table 4 that our proposed CF solver are better than or equal to those reported results. To be more specific, CF solver obtains for 3 problems values of the total intercellular movement that are equal to the best results found in GABB, MIP, and SA methods and improves the values for the rest $6(67 \%)$ problems. It is worth to mention that our proposed CF solver was able to find the optimal solution in 1.547 seconds, illustrating the superiority of CF solver in solution efficiency. 
Table 4. Performance of the proposed approach compared to other approaches

\begin{tabular}{|c|c|c|c|c|c|c|c|c|}
\hline \multicolumn{3}{|c|}{ Test instances } & \multicolumn{3}{|c|}{ Other approaches } & \multicolumn{3}{|c|}{ Proposed approach } \\
\hline \multirow{2}{*}{ No. } & \multirow{2}{*}{$L_{m}$} & \multirow{2}{*}{$U_{m}$} & \multirow{2}{*}{$\begin{array}{c}\text { GABB }[ \\
10] \\
\text { ICM }\end{array}$} & \multirow{2}{*}{$\begin{array}{c}\text { MIP[8] } \\
\text { ICM }\end{array}$} & \multirow{2}{*}{$\begin{array}{c}\text { SA[9] } \\
\text { ICM }\end{array}$} & \multicolumn{3}{|c|}{ CF solver } \\
\hline & & & & & & $\mathrm{NC}$ & ICM & CPU (s) \\
\hline 1 & 2 & 4 & 13 & - & - & 2 & 13 & 0.273 \\
\hline 2 & 2 & 5 & - & - & 29 & 3 & 29 & 0.500 \\
\hline 3 & 2 & 6 & - & 1800 & - & 3 & 1260 & 0.360 \\
\hline 4 & 2 & 5 & - & - & 25 & 3 & 25 & 0.578 \\
\hline 5 & 2 & 6 & - & 34979 & - & 3 & 27416 & 0.907 \\
\hline 6 & 2 & 7 & - & - & 34 & 3 & 32 & 0.774 \\
\hline 7 & 2 & 5 & 18 & - & - & 5 & 17 & 0.953 \\
\hline 8 & 2 & 5 & 86 & - & - & 5 & 82 & 1.789 \\
\hline 9 & 2 & 4 & 39 & - & - & 7 & 33 & 1.547 \\
\hline
\end{tabular}

\section{Conclusions}

Considering alternative process routings and machine sequences to cell formation (CF) problems makes the problems more realistic. New technologies, especially the World-Wide Web (WWW) technologies, have created many opportunities for research about Decision Support Systems (DSS). In this study, a Web-based CF DSS considering alternative process routings and machine sequences simultaneously has been proposed. With the assistance of CF DSS, the CF practitioners in the production departments can interact with the systems without knowing the details of algorithms and can get the best machine cells and part families with minimize the total intercellular movement wherever and whenever they may need it. An example taken from the literature was adopted for illustrational purpose. To further verify the feasibility and effectiveness of the system developed, a set of test problems with various sizes drawn from the literature have be used to test the performance of the CF solver. Corresponding results were compared to several well-known algorithms previously published. The results indicated that the proposed CF solver improved the best results found in the literature for $67 \%$ of the test problems and the CPU times took by our proposed CF solver to find the optimal solution were in 1.547 seconds. These show that our developed system should be very effective, efficient and practical.

\section{REFERENCES}

[1] S. Heragu, "Facilities design," Massachusetts: PWS Publishing Company, Boston, 1997.

[2] U. Wemmerlov and N. Hyer, "Research issues in cellular manufacturing," International Journal of Production Research, Vol. 25, pp. 413-431, 1987.
[3] A. Kusiak, “Intelligent manufacturing systems," New Jersey: Prentice Hall, Englewood Cliffs, 1990.

[4] Y. Yin and K. Yasuda, "Similarity coefficient methods applied to the cell formation problem: A taxonomy and review," International Journal of Production Economics, Vol. 101, pp. 329-352, 2006.

[5] A. Kusiak, "The generalized group technology concept," International Journal of Production Research, Vol. 25, pp. 561-569, 1987.

[6] M. S. J. Ameli and J. Arkat, "Cell formation with alternative process routings and machine reliability consideration,” International Journal of Advanced Manufacturing Technology, Vol. 35, pp. 761-768, 2008.

[7] F. Boctor, "A linear formulation of the machine-part cell formation problem,” International Journal of Production Research, Vol. 29, No. 2, pp. 343-356, 1991.

[8] Y. Y. Won and K. C. Lee, “Group technology cell formation considering operation sequences and production volumes,” International Journal of Production Research, Vol. 39, No. 13, pp. 2755-2768, 2001.

[9] S. Sofianopoulou, "Manufacturing cells design with alternative process plans and/or replicate machines,” International Journal of Production Research, Vol. 37, pp. 707720, 1999.

[10] M. Boulif and K. Atif, “A new branch-\&-bound-enhanced genetic algorithm for the manufacturing cell formation problem,” Computers and Operations Research, Vol. 33, pp. 2219-2245, 2006.

[11] T.-H. Wu, S.-H. Chung, and C.-C. Chang, "Hybrid simulated annealing algorithm with mutation operator to the cell formation problem with alternative process routings," Expert Systems with Applications, Vol. 36, pp. 36523661, 2008.

[12] G. J. Nair and T. T. Narendran, "CASE: A clustering algorithm for cell formation with sequence data," International Journal of Production Research, Vol. 36, pp. 157179, 1998.

[13] J. McAuley, "Machine grouping for efficient production," Production Engineer, Vol. 52, pp. 53-57, 1972.

[14] S. Lozano, B. Adenso-Diaz, and L. Onieva, "A one-step Tabu search algorithm for manufacturing cell design," Journal of the Operational Research Society, Vol. 50, pp. 509-516, 1999.

[15] G. Y. Tian, G. Yin, and D. Taylor, "Internet-based manufacturing: A review and a new infrastructure for distributed intelligent manufacturing," Journal of Intelligent Manufacturing, Vol. 13, No. 5, pp. 323-338, 2002.

[16] J. Lee, "E-manufacturing systems: Fundamental and tools," Robotics and Computer-Integrated Manufacturing, Vol. 9, No. 6, pp. 501-507, 2003.

[17] C. S. Smith and P. K. Wright, "CyberCut: A world-wide web-based design-to-fabrication tool,” Journal of Intelligent Manufacturing, Vol. 15, No. 6, pp. 432-442, 1996.

[18] N. Wu, "A concurrent approach to cell formation and assignment of identical machines in group technology," 
International Journal of Production Research, Vol. 36, pp. 2099-2114, 1998.

[19] T. Gupta and H. Seifoddini, "Production data based similarity coefficient for machine-part grouping decisions in the design of a cellular manufacturing system," International Journal of Production Research, Vol. 28, pp. 1247-
1269, 1990.

[20] R. Nagi, G. Harlarakis, and J. M. Proth, "Multiple routings and capacity considerations in group technology applications,” International Journal of Production Research, Vol. 28, pp. 2243-2257, 1990. 